\title{
Benign prostat hiperplazisi ile ilișkili alt üriner sistem yakınmalarının tedavisinde tadalafil 5 mg'ın etkinliği
}

\author{
Efficacy of tadalafil $5 \mathrm{mg}$ in the treatment of benign prostatic hyperplasia- \\ related lower urinary tract symptoms
}

Ali $\operatorname{Atan}^{1}$

\section{öz}

BPH ile ilişkili bu alt üriner sistem yakınmalarının tedavisinde tadalafil $5 \mathrm{mg}$ son yıllarda klinik kullanıma girmiş medikal tedavi seçeneklerinden bir tanesidir. Bu derlemede tadalafil 5 mg'ın BPH/AÜSY için klinik kullanıma girişi ve etkinliği anlatılmaktadır.

Anahtar Kelimeler: BPH, alt üriner sistem yakınmaları, tadalafil
$\mathbf{P}$ rostatın stroma-glandüler hiperlazisi olan Benign Prostat Hiperplazisi (BPH), erkeklerdeki en sik alt üriner sistem yakınmalarının (AÜSY) nedenidir. BPH ile ilişkili bu alt üriner sistem yakınmalarının (BPH/AÜSY) tedavisinde pek çok seçenek vardır. Yakınmaların ciddiyetine ve rahatsızlık derecesine, hastanın tercihine ve tedaviyi yapacak olan hekimin deneyimine ve teknolojik donanımına göre, bu seçeneklerden tercih yapılır. Hafif yakınma düzeyinde, izlem ve gözlem seçeneği uygulanabilir. Yakınmaların orta-ciddi düzeyde ve rahatsızlık derecesinin fazla olduğu olgularda, öncelikle medikal tedaviler kullanılmaktadır. BPH/AÜSY tedavisinde medikal tedavi olarak, uzun yıllar sadece alfa blokerler ve 5-alfa redüktaz inhibitörü ilaçlar kullanıldı. Alfa blokerler, prostat stromasında bulunan alfa reseptörleri bloke ederek ve düz kas relaksasyonu sağlayarak, 5-alfa redüktaz inhibitörleri ise periuretral adenom kitlesini küçülterek etkinliklerini gösterir. Her iki medikal tedavide de amaç, BPH'ne bağlı meydana gelen mesane çıkım direncini azaltmaktır. Ancak, BPH ile ilgili

${ }^{1}$ Gazi Üniversitesi, Tıp Fakültesi, Üroloji Anabilim Dalı

Yazışma Adresi/ Correspondence:

Prof. Dr. Ali Atan

Birlik Mahallesi, 396 Sok., No: 14/11, Çankaya, Ankara / Türkiye

Tel. $\quad+905324242082$

E-mail: aliatanpitt@hotmail.com

Geliș/Received: $\quad 22.03 .2017$

Kabul/Accepted: $\quad 27.03 .2017$

\section{ABSTRACT}

In the treatment of BPH-related lower urinary tract symptoms, tadalafil $5 \mathrm{mg}$ is of the medical treatment options that have been used clinically in recent years. In this review, introduction to clinical use and efficacy of tadalafil $5 \mathrm{mg}$ for BPH/LUTS is described..

Keywords: BPH, lower urinary tract symptoms, tadalafil

çalışmaların artması sonucunda, BPH/AÜSY'nın ortaya çımasında prostat ile beraber mesanenin de çok önemli olduğu anlaşılmıştır. Bu nedenle, özellikle depolama yakınmaları ön planda olan erkeklerde, anti-muskariniklerin klinik kullanımı başlamıştır. Ayrıca, BPH ile Erektil Disfonksiyon (ED)'un ortak bir fizyopatolojik temele bağlı olarak meydana geldiğinin saptanması sonucu da, fosfodiesteraz Tip 5 enzim inhibitörü (PDE-5i) olan tadalafil 5 mg, BPH tedavisinde klinik kullanıma girmiştir. ${ }^{[1]}$

İlk olarak 2002 yılında, ED tedavisi nedeniyle PDE-5i alan hastalarda rastlantısal olarak AÜSY'larının düzeldiği fark edilmiştir. ${ }^{[2]}$ Daha sonra Mulhall ve arkadaşları, ED nedeniyle sildenafil verdikleri hastalarda AÜSY'nın durumunu incelemişler ve önceki çalışmanın sonucunu teyit etmişlerdir. ${ }^{[3]}$ PDE-5i ilaçların BPH/AÜSY tedavisindeki etkinliği bir kaç nedene bağlıdır. BPH/AÜSY ve ED ortak fizyopatolojik temele dayanmaktadır. Her hastalıkta da, otonom aşırı aktivite, Rho-kinaz aktivite artışı, pelvik ateroskleroz/ iskemi ve pelvik Nitrik Oksid Sentaz / Nitrik Oksid düzeylerinde azalma vardır. ${ }^{[4]}$ BPH/AÜSY, sıklıkla ED ile beraberdir. Her iki durumun sıklığı ve ciddiyeti yaş ile artar. Bir durumun ciddiyeti arttığında, sıklıkla diğer durumun ciddiyeti de artar. Bir durum için tedavi arandığında, aynı hastada her iki durum da mevcuttur. ${ }^{[5,6]}$ Bunlara ek olarak, deneysel çalışmalarda PDE-5 ekspresyonunun üriner sistemin tüm dokularında bulunduğu da gösterilmiştir. Sıklık sırasına göre; mesanede, uretrada, korpus 
kavernozumlarda, prostatta, üreterde ve böbrekte PDE-5 ekspresyonu gösterilmiştir. ${ }^{[7]}$ PDE-5i ilaçların, prostat içinde relaksasyon oluşmasına, perfüzyonun restore olmasına ve oksijenizasyonun artmasına, afferent sinir aktivitesinin inhibisyonuna, inflamasyonun azalmasına ve düz kas hücre proliferasyonunun azalmasına (antiproliferatif etki) neden olarak, AÜSY üzerinde etkili olduğu düşünülmektedir. ${ }^{[7-10]}$

BPH/AÜSY tedavisinde PDE-5i ilaçların kullanıldığı ilk çalışmalarda, hem kısa hem uzun etkili ajanlar kullanılmıştır. ${ }^{[11,12]} \mathrm{Bu}$ çalışmalarda, kısa ve uzun etkili PDE-5i ilaçların BPH semptomlarında düzelme sağladığı gösterilmiştir. Ancak, kısa etkili PDE-5i ilaçların kullanıldığı randomize kontrollü çalışmaların sayısı yetersizdir ve bu moleküllerin BPH/AÜSY tedavisinde kullanımları ile ilgili FDA onaylar1 yoktur. ${ }^{[13,14]} \mathrm{Bu}$ nedenle, günümüzde BPH/AÜSY tedavisinde kullanım onayı olan tek ilaç tadalafil 5 mg'dır.

BPH ile ilişkili AÜSY tedavisinde PDE-5i kullanımı ile ilgili ilk sistemik derlemede, 2007 ve 2008 yıllarına ait dört çalışma incelenmiştir. Bu çalışmalara toplam 1928 hasta alınmıştır. Bu derleme makalede, PDE-5i ile, Uluslararası Prostat Semptom Skoru (IPSS) ve hayat kalitesi skoru ile Uluslararası Erektil Fonksiyon İndeksi (IIEF) değerlerinin anlamlı düzeldiği, ancak maksimum idrar akımı (Qmax) değişiminin anlamlı olmadığı saptanmıştır. ${ }^{[11]}$ Daha sonra, 2011 yılında Liu ve arkadaşlarının yaptığı sistemik bir derleme ve meta-analiz çalışmasında, IPSS, Qmax ve postvoiding rezidüel idrar (PVR) miktarı üzerinde PDE$5 \mathrm{i}$ ilaçların etkisi incelendi. Bu derlemede IPSS düzelmesi, tadalafil ve plasebo için 5 ve 2,7 , vardenafil ve plasebo için 5,8 ve 3,6 , sildenafil ve plasebo için 6,3 ve 1,9 puan olarak saptandı. Tüm PDE-5i ilaçlar ile plasebo, IPSS değişimi 5,24 ve 2,64 puan olarak bulundu. Ancak, önceki derlemede olduğu gibi Qmax değerinde anlamlı bir artış olmadı ve PVR düzeyi değişim göstermedi. Yazarlar, ED ve BPH/AÜSY'da ilk tercihin PDE-5i ilaçlar olduğunu belirtmişlerdir. ${ }^{[12]}$ Gacci ve arkadaşlarının derlemesinde, BPH/AÜSY tedavisinde PDE-5i ilaçların tek başına ve alfa blokerler ile kombine kullanımının etkinliği incelenmiştir. Yazarlar, plaseboya göre PDE-5i ajanlar ile, IIEF, IPSS, hayat kalitesinin anlamlı düzeldiğini, ancak Qmax düzeyinde anlamlı bir artışın olmadığını ve etkinliğin ED olan ve olmayan hastalarda aynı olduğunu saptamışlardır. Ancak, alfa bloker ve PDE-5i ilaç kombinasyonu ile sadece alfa bloker kullanımının etkinliği karşılaştırıldığında, IPSS, IIEF ve Qmax değerlerinde anlamlı düzelmenin olduğunu göstermişlerdir. ${ }^{[14]}$ Yedi çalışmanın dahil edildiği başka bir meta-analizde, alfa bloker ve PDE-5i kombinasyonu ile sadece PDE-5i ilaçların kullanımı karşılaşıııılmıştır. Kombinasyon grubunda, IPSS, IIEF ve Qmax düzeylerinde anlamlı düzelme gösterilmiştir. ${ }^{[15]}$
BPH/AÜSY tedavisinde PDE-5i ilaçların, IIEF, IPSS ve hayat kalitesi üzerindeki etkisi pek çok çalışmada gösterilmiş olmasına karşın, maksimum idrar akımı üzerindeki etkisi değişik çalışmalarda farklılık göstermektedir. Toplam 16 randomize, çift kör ve plasebo kontrollü çalışmanın (14 tadalafil, 1 sildenafil, 1 vardenafil) dahil olduğu başka bir derlemede, PDE5-i ilaçların total IPSS, BPH etki indeksi ve IIEF üzerinde anlamı düzelme sağladıkları, IPSS üzerindeki etkilerinin hem depolama hem de miksiyon yakınmaları için geçerli olduğu, ED varlığı veya yokluğunun tadalafilin IPSS üzerindeki etkisi için önemli olmadığı saptanmıştır. Ayrıca, tadalafil 5 mg'ın, diğer PDE-5i ilaçlardan farklı olarak, Qmax üzerinde anlamlı artış sağladığı görülmüştür. ${ }^{[16]}$ Roehrborn ve arkadaşlarının çalışmasında da, tadalafil 5 mg ile Qmax artışı gösterilmiştir. ${ }^{[17]}$ Ayrıca, ereksiyon bozukluğunun varlığının veya yokluğunun da tadalafilin etkisini değiştirmediği saptanmıştır. ${ }^{[18]}$ Chapple ve arkadaşlarının bir derleme çalışmasında ise, tadalafil 5 mg'ın depolama ve miksiyon yakınmaları üzerindeki etkisi incelenmiştir. Bu çalışmada, tadalafil 5 mg'ın 12 haftalık kullanımda depolama ve miksiyon yakınmaları üzerinde benzer etki yaptığı gösterilmiştir. Yüksek ve düşük depolama yakınması varlığında tadalafilin etkisinin aynı olduğu bulunmuştur. ${ }^{[19]}$

Oelke ve arkadaşlarının bir çalışmasında, tadalafil 5 mg'ın etkinliğinin başlama zamanı incelenmiştir. Bu çalışmada. tadalafil 5 mg' in 12 hafta kullanıldığı, dört plasebo kontrollü, çift kör çalışmaya alınan 1477 hastanın verileri incelenmiştir. $\mathrm{Bu}$ analizde, hastaların yarısından fazlasında BPH/AÜSY düzelmesinin ilk haftanın sonunda olduğu, dört haftalık kullanımda ise hastaların \%70'ten fazlasın$\mathrm{da}$ etkinliğin görüldüğü belirtilmektedir. ${ }^{[20]} \mathrm{Bu}$ veriler 1şığında, tadalafil $5 \mathrm{mg}$ tedavisinin BPH/AÜSY tedavisindeki etkisinin bir hafta gibi çok erken sürede başladığını söyleyebiliriz.

BPH/AÜSY olan kişilerin PDE-5i için uygun aday olup olmadıkları konusu araştırıcılar tarafından merak edilmiştir. Bu konunun incelendiği bir çalışmada, başlangıçtaki AÜSY ciddiyetinin $(<20,20 \geq)$, yaşın $(\leq 65,>65$ yll $)$, önceden alfa adrenerjik ilaç veya PDE-5i kullanım öyküsünün varlığının, total testosteron düzeyinin $(<300,300 \geq \mu \mathrm{g} / \mathrm{dL})$, PSA ile öngörülen prostat volümünün $(\leq 40,>40 \mathrm{~mL})$, hipertansiyon, diabetes mellitus, kardiyovasküler hastalık varlığı veya yokluğunun önemli olmadığı saptanmıştır. ${ }^{[21]}$ Yani, BPH/AÜSY olan her hasta bu tedavi için uygun bir adaydır. Ancak, PDE-5i kullanımı ile ilgili bir kontrendikasyonun olmaması gereklidir. PDE5i kullanımının kontrendikasyonları ise; nitrat kullanımı, potasyum kanal açıcısı olan nikorandil kullanımı, selektif olmayan alfa blokerler (doxazosin and terazosin), unstable 
angina pectoris, yeni geçirilmiş miyokard enfarktüsü $(<3$ ay), miyokard yetmezliği (New York Heart Association stage $>2$ ), inme (<6 ay), hipotansiyon, kötü kontrollü kan basıncı, anlamlı KC ve böbrek yetmezliği, önceden PDE-5i kullanımına bağlı ani görme kaybı ile birlikte anterior iskemik optik nöropati varlığıdır. ${ }^{[1]}$

Bir diğer merak konusu ise, uzun süreli kullanım sonrası PDE-5i ilaçların etkinliğinin devam edip etmediğidir. Tadalafil 5 mg'in BPH/AÜSY tedavisindeki en uzun kullanımı 52 haftadır. Bu sürede, hastalar bu tedaviden fayda görmüşlerdir. ${ }^{[22]}$ Ancak, daha uzun süreli kullanım konusunda elimizde veri yoktur.

Sonuç olarak; Tadalafil 5 mg'in AÜSY monoterapisinde yeri vardır ve etkinlik 1 . haftada başlamaktadır. Tadalafil $5 \mathrm{mg}$ ile, IPSS'de (depolama-miksiyon), BPH etki indeksinde ve hayat kalitesi skorunda düzelme ile beraber Qmax düzeyinde de artış olmaktadır. Etkinlik için, ED varlığı veya yokluğu önemli değildir. Gerek duyulduğunda alfa blokerler ile kombine kullanım mümkündür. Bir yıl üzeri kullanımda etkinlik ve tolerabilite ile ilgili veri yoktur.

\section{KAYNAKLAR}

1. Gravas S, Bach T, Bachmann A, et al. EAU Guidelines on the Management of non-Neurogenic Male Lower Urinary Tract Symptoms (LUTS), incl. Benign Prostatic Obstruction (BPO) European Association of Urology 2015.

2. Sairam K, Kulinskaya E, McNicholas TA, et al. Sildenafil influences lower urinary tract symptoms. BJU Int 2002;90:836-9.

3. Mulhall JP, Guhring P, Parker M, Hopps C. Assessment of the impact of sildenafil citrate on lower urinary tract symptoms in men with erectile dysfunction. J Sex Med 2006;3:662-7. doi: 10.1111/j.1743-6109.2006.00259.x

4. Govorov A, Kasyan G, Priymak D, et al. Tadalafil in the management of lower urinary tract symptoms: a review of the literature and current practices in Russia. Cent European J Urol 2014;67:167-77. doi: 10.5173/ceju.2014.02.art10

5. Seftel AD, de la Rosette J, Birt J, et al. Coexisting lower urinary tract symptoms and erectile dysfunction: a systematic review of epidemiological data. Int J Clin Pract 2013;67:32-45. doi: 10.1111/ijcp. 12044

6. Rosen R, Altwein J, Boyle P, et al. Lower urinary tract symptoms and male sexual dysfunction: the multinational survey of the aging male (MSAM-7). Eur Urol 2003;44:637-49.

7. Tinel H, Stelte-Ludwig B, Hütter J, Sandner P. Pre-clinical evidence for the use of phosphodiesterase- 5 inhibitors for treating benign prostatic hyperplasia and lower urinary tract symptoms. BJU Int 2006;96:1259-63. doi: 10.1111/j.1464-410X.2006.06501.x

8. Filippi S, Morelli A, Sandner P, et al. Characterization and functional role of androgen-dependent PDE5 activity in the bladder. Endocrinology 2007;148:1019-29. doi: 10.1210/ en.2006-1079

9. Andersson KE, de Groat WC, McVary KT, et al. Tadalafil for the treatment of lower urinary tract symptoms secondary to benign prostatic hyperplasia: pathophysiology and mechanism(s) of action. Neurourol Urodyn 2011;30:292-301. doi: 10.1002/nau.20999
10. Gacci M, Andersson KE, Chapple C, et al. Latest Evidence on the Use of Phosphodiesterase Type 5 Inhibitors for the Treatment of Lower Urinary Tract Symptoms Secondary to Benign Prostatic Hyperplasia. Eur Urol 2016;70:124-33. doi: 10.1016/j. eururo. 2015

11. Laydner HK, Oliveira P, Oliveira CR, et al. Phosphodiesterase 5 inhibitors for lower urinary tract symptoms secondary to benign prostatic hyperplasia: a systematic review. BJU Int 2010;107:11049. doi: 10.1111/j.1464-410X.2010.09698.x

12. Liu L, Zheng S, Han P, Wei Q. Phosphodiesterase-5 inhibitors for lower urinary tract symptoms secondary to benign prostatic hyperplasia: a systematic review and meta-analysis. Urology 2011;77:123-9. doi: 10.1016/j.urology.2010.07.508

13. McVary KT, Monnig W, Camps JL Jr, et al. Sildenafil citrate improves erectile function and urinary symptoms in men with erectile dysfunction and lower urinary tract symptoms associated with benign prostatic hyperplasia: a randomized, double-blind trial. J Urol 2007;77:1071-7. doi: 10.1016/j.juro.2006.10.055

14. Gacci M, Vittori G, Tosi $\mathrm{N}$, et al. A randomized, placebocontrolled study to assess safety and efficacy of vardenafil $10 \mathrm{mg}$ and tamsulosin $0.4 \mathrm{mg}$ vs. tamsulosin $0.4 \mathrm{mg}$ alone in the treatment of lower urinary tract symptoms secondary to benign prostatic hyperplasia. J Sex Med 2012;9:1624-33. doi: 10.1111/j.17436109.2012.02718.x

15. Yan H, Zong H, Cui Y, Li N, Zhang Y. The efficacy of PDE5 inhibitors alone or in combination with alpha-blockers for the treatment of erectile dysfunction and lower urinary tract symptoms due to benign prostatic hyperplasia: a systematic review and metaanalysis. J Sex Med 2014;11:1539-45. doi: 10.1111/jsm.12499

16. Zhang LT, Park JK. Are phosphodiesterase type 5 inhibitors effective for the management of lower urinary symptoms suggestive of benign prostatic hyperplasia? World J Nephrol 2015;4:138-47. doi: $10.5527 /$ wjn.v4.i1.138

17. Roehrborn CG, Chapple C, Oelke M, Cox D, Esler A, Viktrup L. Effects of tadalafil once daily on maximum urinary flow rate in men with lower urinary tract symptoms suggestive of benign prostatic hyperplasia. J Urol 2014;191:1045-50. doi: 10.1016/j. juro.2013.10.074

18. Hatzimouratidis K. A review of the use of tadalafil in the treatment of benign prostatic hyperplasia in men with and without erectile dysfunction. Ther Adv Urol 2014;6:135-147. doi: $10.1177 / 1756287214531639$

19. Chapple CR, Roehrborn CG, McVary K, Ilo D, Henneges C, Viktrup L. Effect of tadalafil on male lower urinary tract symptoms: an integrated analysis of storage and voiding international prostate symptom subscores from four randomised controlled trials. Eur Urol 2015;67:114-22. doi: 10.1016/j.eururo.2014.08.072

20. Oelke M, Shinghal R, Sontag A, Baygani SK, Donatucci CF. Time to onset of clinically meaningful improvement with tadalafil $5 \mathrm{mg}$ once daily for lower urinary tract symptoms secondary to benign prostatic hyperplasia: analysis of data pooled from 4 pivotal, double-blind, placebo controlled studies. J Urol 2015;193:15819. doi: 10.1016/j.juro.2014.11.094

21. Porst H, Oelke M, Goldfischer ER, et al. Efficacy and safety of tadalafil $5 \mathrm{mg}$ once daily for lower urinary tract symptoms suggestive of benign prostatic hyperplasia: subgroup analyses of pooled data from 4 multinational, randomized, placebo-controlled clinical studies. Urology 2013;82:667-73. doi: 10.1016/j. urology.2013.05.005

22. Donatucci CF, Brock GB, Goldfischer ER, et al. Tadalafil administered once daily for lower urinary tract symptoms secondary to benign prostatic hyperplasia:a 1-year, open-label extension study. BJU Int 2011; 107:1110-6. doi: 10.1111/j.1464410X.2010.09687.x 